\title{
Serum level of soluble CD14 subtype predicts long-term prognosis in sepsis patients with cardiac dysfunction
}

\author{
Junliang Liu ${ }^{1}$, Mingying Dai ${ }^{2}$, Hongyun Yang ${ }^{3}$, Lei Song ${ }^{1}$, Kun Chen ${ }^{4}$, Yanli Wang ${ }^{4}$, Ming Hua ${ }^{4}$ \\ ${ }^{1}$ ICU, Renmin Hospital of Chengyang District, Qingdao, China; ${ }^{2}$ ICU, Affiliated Hospital of Qingdao University, Qingdao, China; ${ }^{3}$ ICU, General \\ Hospital of Eastern Theater, Nanjing, China; ${ }^{4} \mathrm{ICU}$, Guannan Division, The First Renmin Hospital, Lianyungang, China \\ Contributions: (I) Conception and design: J Liu; (II) Administrative support: M Dai; (III) Provision of study materials or patients: H Yang, L Song; (IV) \\ Collection and assembly of data: K Chen, Y Wang; (V) Data analysis and interpretation: J Liu, M Hua; (VI) Manuscript writing: All authors; (VII) \\ Final approval of manuscript: All authors. \\ Correspondence to: Ming Hua. ICU, Guannan Division, The First Renmin Hospital, Lianyungang 222042, China. Email: hm2000lyg@163.com.
}

Background: Sepsis is defined as a life-threatening organ dysfunction caused by a dysregulated host response to infection, and some sepsis patients will develop cardiac dysfunction. Sepsis-induced cardiac dysfunction (SICD) has been demonstrated to be a promising predictor of mortality, although the prediction of SICD itself remains unclear. Clinical studies have shown that soluble CD14 subtype (sCD14-ST) may be a useful predictor for sepsis. In this study, we aimed to evaluate the predictive value of sCD14-ST for SICD in patients with sepsis.

Methods: Patients with SICD from three intensive care units (ICUs) of three medical centers between January 2015 and December 2018 were enrolled. Clinical data and information were collected from hospital and clinic records. Blood samples at admission were collected and serum levels of sCD14-ST were tested. Patients were followed up for at least 1 year. Major adverse cardiovascular events (MACEs) were recorded. Echocardiography was repeated at the end of 1-year follow-up.

Results: A total of 117 patients were enrolled into the final analysis. During 1-year follow-up, MACEs occurred in 35 (29.9\%) patients. Most MACEs occurred with 3 months after discharge. Univariate and multivariate analysis revealed that age $(\mathrm{OR}=1.5,95 \% \mathrm{CI}: 1.2-2.3, \mathrm{P}=0.036)$, cardiac troponin $\mathrm{T}$ (cTnT) ( $\mathrm{OR}=1.4,95 \% \mathrm{CI}: 1.2-2.1, \mathrm{P}=0.027)$, creatine $(\mathrm{Cr})(\mathrm{OR}=1.6,95 \% \mathrm{CI}: 1.3-2.5, \mathrm{P}=0.022)$, sequential organ failure assessment (SOFA) score $(\mathrm{OR}=1.7,95 \% \mathrm{CI}: 1.3-2.6, \mathrm{P}=0.012)$, and soluble cluster of differentiation 14 subtype (sCD14-ST) (OR =1.9, 95\% CI:1.4-3.1, P=0.015) were predictors for MACEs in patients with SICD at 1-year follow-up. Area under receiver operating curve (AUROC) of sCD14-ST to MACEs was 0.784 , and the cutoff point was $748.3 \mu \mathrm{g} / \mathrm{L}$ with a sensitivity of 0.78 and a specificity of 0.74 respectively. Blood test at the end of 1-year follow-up revealed that patients with a lower sCD14-ST level had better lower Cr, N-terminal pro-brain natriuretic peptide (NT-proBNP) and higher systolic blood pressure (SBP) and left ventricular ejection fraction (LVEF).

Conclusions: MACEs mainly occurred within 3 months after discharge in patients with SICD, and high baseline serum levels of sCD14-ST predicted poor prognosis in patients with SICD.

Keywords: Sepsis; soluble CD14 subtype (sCD14-ST); sepsis induced cardiac dysfunction; prognosis

Submitted Apr 20, 2020. Accepted for publication Jun 29, 2020.

doi: 10.21037/apm-20-1093

View this article at: http://dx.doi.org/10.21037/apm-20-1093 


\section{Introduction}

Sepsis is defined as life-threatening organ dysfunction caused by a dysregulated host response to infection (1). Previous research has mainly focused on infectious inflammation, shock, renal dysfunction, etc. In recent years, a greater number of studies have begun to identify the importance of sepsis on myocardia and heart function. Some phenomena that have been linked with sepsisrelated cardiac damage include septic cardiomyopathy, sepsis-induced myocardial dysfunction, sepsis-induced cardiac dysfunction (SICD), and others. In fact, these terms refer to the same thing: heart injury secondary to severe infection resulting in cardiac dysfunction. Two points here are important for the definition: first, heart injury and cardiac dysfunction must happen after infection; second, there can be no other identifiable cause for these cardiac problems. In our study, we use the term "SICD" to emphasize the basic pathophysiological process and characteristic of this phenomenon. One characteristic of SICD is that left ventricular dysfunction is reversible. In clinical experience, when the patient's condition involves an infection that has been effectively controlled with septic shock being resolved, cardiac function can recover in turn (2). In ICU, present assessment about SICD mainly includes electrocardiogram, pulse index continuous pardiac output (PiCCO), echocardiography, biomarkers including cardiac troponin $\mathrm{T} / \mathrm{I}(\mathrm{c} T \mathrm{~T} \mathrm{~T} / \mathrm{I})$ and $\mathrm{N}$-terminal pro-brain natriuretic peptide (NT-proBNP). Specific treatment includes cardiopulmonary support and medication including trimetazidine, fasudil hydrochloride, levosimendan and esmolol. However, other clinical observational research has demonstrated that cardiac dysfunction may be a risk factor for poor prognosis in medium- to long-term followup (3). Otherwise, how to predict the long-term prognosis of patients with SICD remains unclear. In recent years, soluble cluster of differentiation 14 subtype (sCD14-ST) has gained significant attention as a useful predictor of patients suffering sepsis. Previous experimental studies have demonstrated that the biological role of sCD14-ST is very complicated $(4,5)$. Based on these previous studies, in this study, we aimed to evaluate the predictive value of sCD14$\mathrm{ST}$ in the long-term prognosis in patients specifically with SICD. We present the following article in accordance with the STROBE reporting checklist (available at http://dx.doi. org/10.21037/apm-20-1093).

\section{Methods}

\section{Study population}

Patients with SICD from three intensive care units (ICUs) of three medical centers between January 2015 and December 2018 were enrolled. The inclusion criteria for patients were the following: age over 18 years old, meeting sepsis-3 criteria, echocardiography revealing a left ventricular ejection fraction (LVEF) less than 50\%, dilation of left ventricular end-diastolic dimension (LVEDD), elevation of $\mathrm{N}$-terminal pro-brain natriuretic peptide (NTproBNP), and elevation of cardiac troponin $\mathrm{T}$ (c $\mathrm{Tn} \mathrm{T})$. Meanwhile, the exclusion criteria were the following: history of confirmed unstable coronary heart disease (unstable angina pectoris, acute myocardial infarction) within 1 month before admission to the ICU, congenital heart disease, primary or secondary myocardiopathy, or pulmonary heart disease and atrial fibrillation; history of cardiac and pulmonary resuscitation; history of severe renal failure; rheumatic disease; history of chemotherapy or radiotherapy; malignant tumor; and life expectancy less than 1 month. The criteria of left ventricular dysfunction were a serum level of NT-proBNP $\geq 125 \mathrm{ng} / \mathrm{L}$ or $\mathrm{LVEF}<50 \%$. Severe renal failure was defined as an estimated glomerular filtration rate (eGFR) lower than $60 \mathrm{~mL} / \mathrm{min} \cdot \mathrm{m}^{2}$. All patients signed informed content. The study was conducted in accordance with the Declaration of Helsinki (as revised in 2013) and was approved by the ethics committee of our hospital (Lot. 20140704).

\section{Definition of SICD}

The most commonly accepted definition of SICD is reversible myocardial dysfunction caused by sepsis. There are three main features: an LVEF less than $50 \%$ as the diagnostic criterion, left ventricular dilatation, and a return to a normal clinical condition during the early stage of the disease (D). The sequential organ failure assessment (SOFA) score of each patient enrolled in the final analysis was performed at the time of admission or according to clinical record.

\section{Blood test}

Blood samples were taken via direct venous puncture into 
Table 1 Baseline characteristics $(\mathrm{n}=117)$

\begin{tabular}{|c|c|}
\hline Characteristics & Value \\
\hline Age (years) & $50.2 \pm 15.7$ \\
\hline Male $(n, \%)$ & $86(73.5)$ \\
\hline BMI $\left(\mathrm{kg} / \mathrm{m}^{2}\right)$ & $23.7 \pm 4.9$ \\
\hline Surgery within 1 month (n, \%) & $63(53.8)$ \\
\hline \multicolumn{2}{|l|}{ History (n, \%) } \\
\hline Hypertension & $19(16.2)$ \\
\hline Hyperlipidemia & $27(23.1)$ \\
\hline $\mathrm{CHD}$ & $8(6.8)$ \\
\hline DM & $9(7.7)$ \\
\hline COPD & $6(5.1)$ \\
\hline CKD & $4(3.4)$ \\
\hline WBC & $8.3 \pm 5.2$ \\
\hline PCT & $4.2 \pm 3.7$ \\
\hline Bacteria & $93(79.5)$ \\
\hline Fungi & $11(9.4)$ \\
\hline Mixed infection & $13(11.1)$ \\
\hline \multicolumn{2}{|l|}{ cTnT, (n, \%) } \\
\hline Normal & $53(45.3)$ \\
\hline Elevated & $64(54.7)$ \\
\hline NT-proBNP & $3,286 \pm 2,019$ \\
\hline $\mathrm{Cr}$ & $87.4 \pm 30.8$ \\
\hline ALT & $36.9 \pm 17.2$ \\
\hline Fast glucose & $6.4 \pm 2.7$ \\
\hline sCD14-ST & $892.5 \pm 607.4$ \\
\hline SBP & $106.2 \pm 28.5$ \\
\hline LVEF & $45.3 \pm 8.4$ \\
\hline SOFA score & $10.1 \pm 6.9$ \\
\hline
\end{tabular}

$\mathrm{BMI}$, body mass index; $\mathrm{CHD}$, coronary heart disease; $\mathrm{DM}$, diabetes mellitus; COPD, chronic obstructive pulmonary disease; CKD, chronic kidney disease; WBC, white blood cell; PCT, procalcitonin; cTnT, cardiac troponin T; NT-proBNP, N-terminal pro-brain natrium peptide; Cr, creatine; ALT, alanine aminotransferase; sCD14-ST, soluble cluster differentiation 14 subtype; SBP, systolic blood pressure; LVEF, left ventricular ejection fraction; SOFA, sequential organ failure assessment.

tubes containing $10 \mathrm{~mL}$ of sodium citrate on admission. Plasma was isolated within 4 hours after collection of whole blood by centrifugation at $1,800 \times g$ for $10 \mathrm{~min}$ at room temperature. Serum level of sCD14-ST was tested by the PATHFAST Presepsin chemiluminescent enzyme immunoassay (Mitsubishi Chemical Medience Corporation, Japan) using Magtration Technology according to the manufacturer's instructions. A level of sCD14-ST >200 $\mu \mathrm{g} / \mathrm{L}$ was considered positive.

\section{Follow-up}

After discharge from hospital with a stable condition, all enrolled patients were followed up for at least 1 year or until the occurrence of endpoints. Endpoints were considered to be major adverse cardiovascular events, including all-cause death, cardiac death, hospitalization due to heart failure, and stroke.

\section{Statistical analysis}

Continuous variables were expressed as mean \pm standard deviation (SD) and compared using an unpaired twosided Student's $t$-test when normal distribution and equal dispersion were confirmed. The Mann-Whitney $U$ test and Wilcoxon's signed-rank test were used when the variance was unequal. Categorical variables were expressed as percentages (\%) and compared using the $\mathrm{X}^{2}$ analysis or Fisher's exact test if necessary. A P value $<0.05$ was considered statistically significant. All statistical analyses were performed with SPSS (version 17.0 for Windows, SPSS, Inc., Chicago, IL, USA).

\section{Results}

\section{Baseline characteristics}

According to inclusion and exclusion criteria, a total of 117 patients with SICD were enrolled into the final analysis. The age of patients ranged from 24 to 71 years (IQR: 46, average $50.2 \pm 15.7)$. The majority were male $(86,73.5 \%)$. Over half $(53.8 \%)$ of these patients underwent surgery within 1 month before admission to the ICU. Serum levels of sCD14-ST ranged from 149.2 to 2,073.6 $\mu \mathrm{g} / \mathrm{L}$ with an average level of $892.5 \mu \mathrm{g} / \mathrm{L}$. Details were listed in Table 1.

\section{One-year follow-up}

At 1-year follow-up, major adverse cardiovascular events (MACEs) had occurred in 35 (29.9\%) patients (Table 2). A total of 11 (9.4\%) patients died during follow-up with 
Table 2 MACEs at 1-year follow-up

\begin{tabular}{lc}
\hline MACEs & Number (\%) \\
\hline Total & $35(29.9)$ \\
All-cause mortality & $11(9.4)$ \\
Cardiac mortality & $9(6.8)$ \\
Hospitalization due to heart failure & $19(16.2)$ \\
Acute coronary syndrome & $3(2.6)$ \\
Stroke & $2(1.7)$
\end{tabular}

MACEs, major adverse cardiovascular events.

8 (6.8\%) dying within 3 months after discharge. Meanwhile, $9(7.7 \%)$ patients died of cardiac arrest or acute exacerbation of heart failure, and 7 (6.0\%) of them died within 3 months after discharge. A total of 19 (16.2\%) patients were admitted to hospital due to severe heart failure, and 14 (12.0\%) of them were admitted within 3 months after discharge. The other $5(4.3 \%)$ patients were admitted between the $4^{\text {th }}$ month and $10^{\text {th }}$ month after discharge. Acute coronary syndrome occurred in $3(2.6 \%)$ patients between the $5^{\text {th }}$ month and $11^{\text {th }}$ month after discharge, while $2(1.7 \%)$ cases of stroke all happened 7 months after discharge. We further divided the patients into two groups: MACE patients $(n=49)$ and MACE-free patients. Comparison of baseline characteristics between these two groups are listed in Table 3. Patients who suffered MACEs were older. Levels of cTnT, NT-proBNP, creatine (Cr), and sCD14-ST in MACE patients were all higher than those in MACE-free patients.

\section{Factors associated with MACEs in patients with SICD at 1-year follow-up}

Multivariate analysis revealed that multiple factors (including age, cTnT, Cr, SOFA score, sCD14-ST) were associated with MACEs in patients with SICD at 1-year follow-up (Table 4). In this study, we found that elevation of sCD14-ST increased the risk of MACEs at 1-year follow-up with an odds ratio (OR) of 1.9 (95\% CI: 1.4-3.1, $\mathrm{P}=0.015)$. We further calculated the sensitivity and specificity of sCD14-ST to MACEs and found that the area under receiver operating curve (AUROC) was 0.784 , and the cutoff point to discriminate patients at high risk of poor prognosis was $748.3 \mu \mathrm{g} / \mathrm{L}$ with a sensitivity of 0.78 and a specificity of 0.74 (Figure 1).
Table 3 Comparison of baseline characteristics between MACE patients and MACE-free patients

\begin{tabular}{|c|c|c|c|}
\hline Characteristics & $\begin{array}{c}\text { MACE patients } \\
\quad(\mathrm{n}=35)\end{array}$ & $\begin{array}{c}\text { MACE-free } \\
\text { patients }(n=82)\end{array}$ & $P$ value \\
\hline Age & $55.6 \pm 13.2$ & $47.9 \pm 16.9$ & 0.018 \\
\hline Male, (n, \%) & $21(60.0)$ & 65 (79.3) & \\
\hline BMl $\left(\mathrm{kg} / \mathrm{m}^{2}\right)$ & $24.1 \pm 6.1$ & $23.5 \pm 5.7$ & 0.611 \\
\hline Surgery history (n, \%) & $20(57.1)$ & $43(52.4)$ & \\
\hline \multicolumn{4}{|l|}{ History (n, \%) } \\
\hline Hypertension & $5(14.3)$ & $14(17.1)$ & 0.708 \\
\hline Hyperlipidemia & $9(25.7)$ & $18(22.0)$ & 0.658 \\
\hline $\mathrm{CHD}$ & $3(8.6)$ & $5(6.1)$ & 0.932 \\
\hline DM & $3(8.6)$ & $6(7.3)$ & 0.884 \\
\hline COPD & $2(5.7)$ & $4(4.9)$ & 0.787 \\
\hline CKD & $3(8.6)$ & $1(1.2)$ & 0.148 \\
\hline WBC & $7.2 \pm 3.1$ & $8.4 \pm 5.5$ & 0.229 \\
\hline PCT & $5.6 \pm 4.1$ & $3.6 \pm 2.7$ & 0.002 \\
\hline Infection & & & 0.005 \\
\hline Bacteria & 23 & 70 & \\
\hline Fungi & 3 & 8 & \\
\hline Mixed infection & 9 & 4 & \\
\hline cTnT & & & 0.018 \\
\hline Normal & 10 & 43 & \\
\hline Elevated & 25 & 39 & \\
\hline NT-proBNP & $3537 \pm 2481$ & $2923 \pm 1875$ & 0.394 \\
\hline $\mathrm{Cr}$ & $99.3 \pm 41.5$ & $82.3 \pm 23.7$ & 0.006 \\
\hline ALT & $41.7 \pm 20.8$ & $34.9 \pm 19.4$ & 0.092 \\
\hline Fast glucose & $6.8 \pm 3.1$ & $6.2 \pm 2.2$ & 0.237 \\
\hline sCD14-ST & $1307.2 \pm 418.3$ & $715.5 \pm 338.6$ & $<0.001$ \\
\hline SBP & $92.5 \pm 13.7$ & $112.0 \pm 15.6$ & $<0.001$ \\
\hline LVEF & $43.1 \pm 6.5$ & $46.2 \pm 6.1$ & 0.015 \\
\hline SOFA score & $15.4 \pm 7.9$ & $7.8 \pm 5.1$ & $<0.001$ \\
\hline
\end{tabular}

MACE, major adverse cardiovascular event; BMI, body mass index; CHD, coronary heart disease; DM, diabetes mellitus; COPD, chronic obstructive pulmonary disease; CKD, chronic kidney disease; WBC, white blood cell; PCT, procalcitonin; CTnT, cardiac troponin $\mathrm{T}$; NT-proBNP, N-terminal pro-brain natrium peptide; Cr, creatine; ALT, alanine aminotransferase; sCD14-ST, soluble cluster differentiation 14 subtype; SBP, systolic blood pressure; LVEF, left ventricular ejection fraction; SOFA, sequential organ failure assessment. 
Table 4 Multivariate analysis of factors associated with MACEs at 1-year follow-up

\begin{tabular}{lccc}
\hline Factors & OR value & $95 \% \mathrm{Cl}$ & P value \\
\hline Age & 1.5 & $1.2-2.3$ & 0.036 \\
cTnT & 1.4 & $1.2-2.1$ & 0.027 \\
Cr & 1.6 & $1.3-2.5$ & 0.022 \\
SOFA score & 1.7 & $1.3-2.6$ & 0.012 \\
sCD14-ST & 1.9 & $1.4-3.1$ & 0.015 \\
\hline
\end{tabular}

MACEs, major adverse cardiovascular events; cTnT, cardiac troponin $\mathrm{T} ; \mathrm{Cr}$, creatine; SOFA, sequential organ failure assessment; sCD14-ST, soluble cluster differentiation 14 subtype.

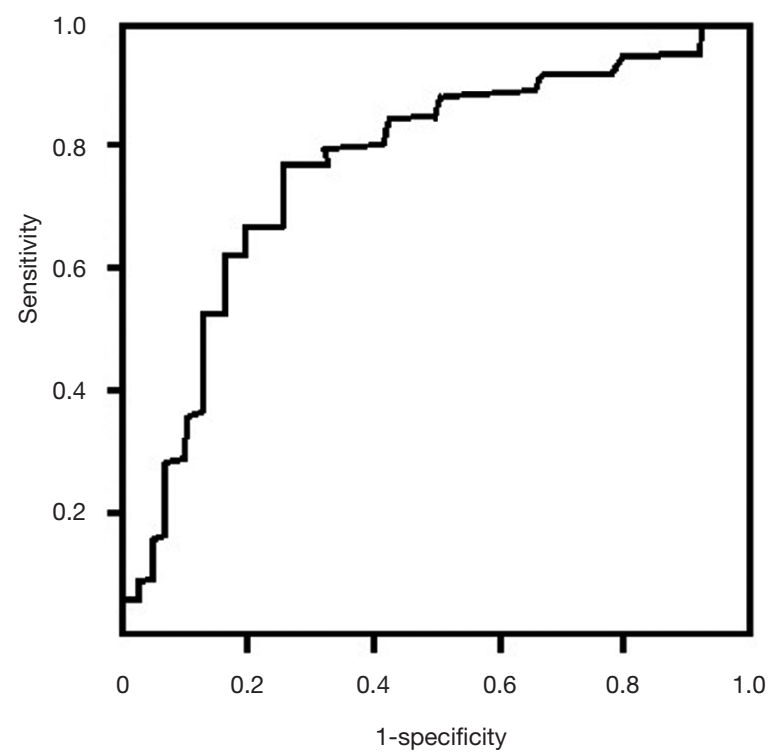

Figure 1 AUC of serum level of sCD14-ST to predict MACEs in patients with sepsis-induced cardiac dysfunction. sCD14-ST, soluble cluster differentiation 14 subtype; MACE, major adverse cardiovascular event.

\section{Prediction of MACEs at 1-year follow-up using baseline serum level of sCD14-ST}

Using the cutoff point of sCD14-ST, we divided the patients into two groups: the high-level $(\geq 748.3 \mu \mathrm{g} / \mathrm{L})$ sCD14-ST group $(\mathrm{n}=62)$ and the low-level $(<748.3 \mu \mathrm{g} / \mathrm{L})$ sCD14-ST group $(n=55)$. A comparison of baseline characteristics showed that patients with a lower serum level of sCD14-ST were younger. These patients also had lower white blood cell count (WBC), procalcitonin (PCT), cTnT, $\mathrm{Cr}$, systolic blood pressure (SBP), and SOFA scores, and higher LVEF. One-year follow-up revealed that patients with a lower serum level of sCD14-ST had fewer MACEs. A blood test at the end of 1-year follow-up revealed that patients with lower sCD14-ST levels had lower Cr and NTproBNP, and higher SBP and LVEF. Details are listed in Table 5. Kaplan-Meier survival curves revealed that patients with a lower serum level of sCD14-ST had better prognosis at 1-year follow-up (Figure 2).

\section{Discussion}

In this study, we retrospectively analyzed clinical and follow-up data from discharged patients who suffered SICD and found that the baseline serum level of sCD14-ST correlated with 1-year prognosis. Multivariate analysis revealed that a high baseline serum level of sCD14-ST was a predictor of poor prognosis at 1-year follow-up. Patients with a lower serum level of sCD14-ST had less MACEs.

With an increasing focus from researchers on the influence of sepsis on the heart, studies concerning SICD (or injury) have increased significantly in quantity. These studies have mainly focused on three aspects: the pathophysiological mechanism of sepsis-induced cardiac injury and dysfunction, the influence of SICD on patients' prognosis, the preventive methods and therapy for this kind of patient. As an adverse complication, SICD is a predictor of short- and long-term poor prognosis in patients with sepsis (6). A previous study revealed that SICD assessed by echocardiography showed that the measurement of mitral annular plane systolic excursion (MAPSE) when combined with APACHE II was a good predictor of mortality. Among the echocardiographic parameters, MAPSE alone was also a good predictor of mortality. However, the author of this study thought that the results required further validation from a larger study (7). As for the pathophysiological mechanism of sepsis-induced cardiac injury and dysfunction, recent studies have suggested that SICD may arise from an interaction of many factors, including inflammation, metabolism, and neuroimmunomodulation (8). This pathological process involves multiple signal transduction pathways $(9,10)$ and a variety of cell types including cardiomyocytes, cardiac fibroblasts, and endothelial cells $(3,11)$. In short, the mechanism involved in SICD is very complicated. Unfortunately, no significant progress has been made of late in the prevention and treatment for SICD as the relevant results are conflicting $(12,13)$. For example, in many studies, levosimendan has been demonstrated to 
Table 5 Baseline characteristics in subgroups of different serum levels of sCD14-ST

\begin{tabular}{|c|c|c|c|}
\hline Characteristics & $\begin{array}{c}\text { Low sCD14-ST } \\
\quad(n=55)\end{array}$ & $\begin{array}{l}\text { High sCD14-ST } \\
\quad(n=62)\end{array}$ & $P$ value \\
\hline Age & $47.0 \pm 14.9$ & $53.0 \pm 16.2$ & 0.040 \\
\hline Male, (n, \%) & $40(72.7)$ & $46(74.2)$ & 0.858 \\
\hline $\mathrm{BMI}\left(\mathrm{kg} / \mathrm{m}^{2}\right)$ & $24.3 \pm 5.1$ & $23.2 \pm 4.4$ & 0.213 \\
\hline Surgery history (n, \%) & $33(60.0)$ & $30(48.4)$ & 0.209 \\
\hline \multicolumn{4}{|l|}{ History (n, \%) } \\
\hline Hypertension & 7 (12.7) & $12(19.4)$ & 0.332 \\
\hline Hyperlipidemia & $11(20.0)$ & $16(25.8)$ & 0.457 \\
\hline $\mathrm{CHD}$ & $2(3.6)$ & $6(9.7)$ & 0.355 \\
\hline $\mathrm{DM}$ & $3(5.5)$ & $6(9.7)$ & 0.612 \\
\hline COPD & $2(3.6)$ & $4(6.5)$ & 0.788 \\
\hline CKD & $1(1.8)$ & $4(6.5)$ & 0.436 \\
\hline WBC & $7.0 \pm 4.2$ & $8.9 \pm 5.5$ & 0.040 \\
\hline РCT & $3.1 \pm 2.1$ & $5.2 \pm 4.2$ & 0.001 \\
\hline Infection & & & 0.177 \\
\hline Bacteria & 47 & 46 & \\
\hline Fungi & 5 & 6 & \\
\hline Mixed infection & 3 & 10 & \\
\hline cTnT & & & 0.001 \\
\hline Normal & 34 & 19 & \\
\hline Elevated & 21 & 43 & \\
\hline NT-proBNP & $3609 \pm 2153$ & $2999 \pm 1902$ & 0.106 \\
\hline $\mathrm{Cr}$ & $81.2 \pm 24.9$ & $92.9 \pm 31.4$ & 0.029 \\
\hline ALT & $35.1 \pm 16.2$ & $38.5 \pm 18.5$ & 0.295 \\
\hline Fast glucose & $6.5 \pm 2.9$ & $6.3 \pm 2.6$ & 0.695 \\
\hline SBP & $110.1 \pm 14.2$ & $102.7 \pm 13.6$ & 0.005 \\
\hline LVEF & $47.1 \pm 7.3$ & $43.7 \pm 6.6$ & 0.002 \\
\hline SOFA score & $8.4 \pm 4.3$ & $11.6 \pm 6.4$ & 0.002 \\
\hline MACEs, (n, \%) & $9(16.4)$ & $26(41.9)$ & 0.003 \\
\hline \multicolumn{4}{|l|}{ 1-year follow-up } \\
\hline $\mathrm{Cr}$ & $68.5 \pm 19.2$ & $80.3 \pm 21.6$ & 0.002 \\
\hline NT-proBNP & $438 \pm 221$ & $823 \pm 429$ & $<0.001$ \\
\hline SBP & $116.8 \pm 20.5$ & $107.9 \pm 19.4$ & 0.018 \\
\hline LVEF & $52.9 \pm 6.1$ & $47.2 \pm 5.9$ & $<0.001$ \\
\hline
\end{tabular}

sCD14-ST, soluble cluster differentiation 14 subtype; BMI, body mass index; CHD, coronary heart disease; DM, diabetes mellitus; COPD, chronic obstructive pulmonary disease; CKD, chronic kidney disease; WBC, white blood cell; PCT, procalcitonin; CTnT, cardiac troponin T; NT-proBNP, N-terminal pro-brain natrium peptide; $\mathrm{Cr}$, creatine; ALT, alanine aminotransferase; SBP, systolic blood pressure; LVEF, left ventricular ejection fraction; SOFA, sequential organ failure assessment; MACE, major adverse cardiovascular event.

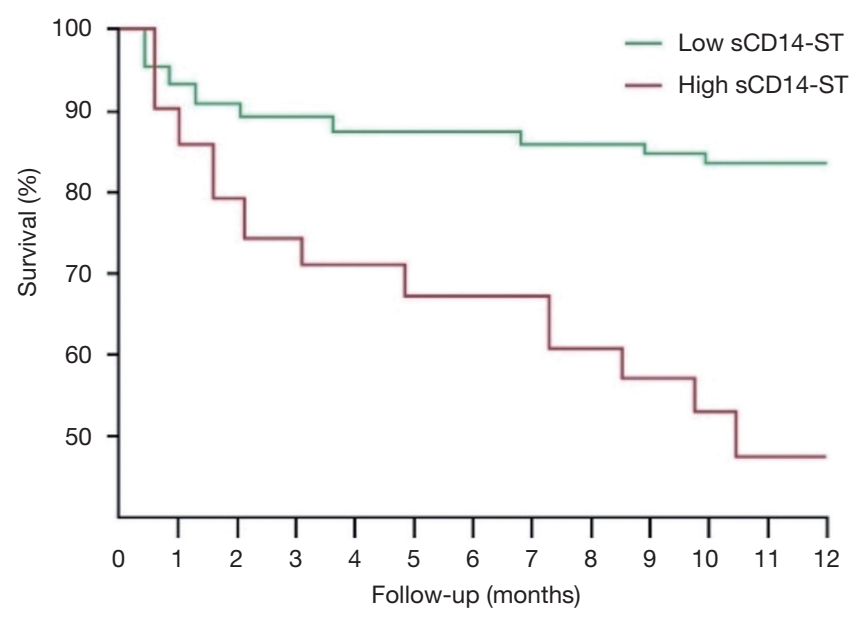

Figure 2 Kaplan-Meier curves of sCD14-ST with MACEs at 1-year follow-up. sCD14-ST, soluble cluster differentiation 14 subtype; MACE, major adverse cardiovascular event.

be useful in treating patients with SICD $(14,15)$, while other studies have found that levosimendan had no effect on patients' prognosis (16) and was even associated with a reduced likelihood of successful extubation and an increased risk of supraventricular tachyarrhythmias (17). Apart from this, in a review of the literature in PubMed and other databases, we found few articles relating to the prediction of prognosis in patients with SICD. Some evidence suggests that patients with SICD can recover if sepsis is cured while others may deteriorate or even die $(18,19)$. It is therefore important to find ways to identify SICD patients who are at risk of poorer prognosis.

Soluble CD14 subtype (sCD14-ST) is the soluble $\mathrm{N}$-terminal region of membrane marker/receptor protein CD14, which is released into the blood flow after host cell activation and follows bacterial lipopolysaccharide (LPS) recognition (20). Previous studies on sCD14-ST mainly focused on its diagnostic role in sepsis $(21,22)$. Research on the prognostic value of sCD14-ST showed that sCD14-ST levels were significantly higher at admission, 24-48 hours after admission, and 7 days after admission in nonsurvivors $v s$. survivors at the same time point (23). In summary, this study suggests that a higher level of sCD14-ST predicted poor short-term prognosis but not specifically for patients with SICD, especially in the long term. In our study, we further investigated the long-term prognostic value of sCD14-ST specifically for patients with SICD. Consistent with findings mentioned above (23), we also found that a higher (cutoff point determined by AUC curve) level of 
sCD14-ST predicted poor prognosis, including in the long term. Repeated blood tests at the end of 1-year follow-up revealed that patients with a lower sCD14-ST level had lower Cr, NT-proBNP, and higher SBP and LVEF. Taken together, it is reasonable to conclude that a higher level of sCD14-ST is associated with poor prognosis.

We also found that the on-admission serum level of sCD14-ST correlated with some other parameters, including WBC, PCT, cTnT, Cr, SBP, SOFA score, and LVEF. This result suggests that sCD14-ST could be used as a risk factor for condition assessment, and this possibility has already been thoroughly investigated $(20,21)$. In this study, we also found that MACEs mainly occurred within 3 months after discharge. Given this, it may be advisable to closely monitor patients within 3 months after discharge in order to discover medical problems as early as possible.

In conclusion, our study suggested that sCD14-ST could be used as a predictor for SICD and that patients with SICD should be under intensive monitoring within 3 months of discharge. Some limitations of this study include relatively smaller sample size, shorter follow-up duration. Further study should be carried out in multiple centers with a larger sample size and a longer follow-up duration.

\section{Acknowledgments}

Funding: None.

\section{Footnote}

Reporting Checklist: The authors have completed the STROBE reporting checklist. Available at http://dx.doi. org/10.21037/apm-20-1093

Data Sharing Statement: Available at http://dx.doi. org/10.21037/apm-20-1093

Conflicts of Interest: All authors have completed the ICMJE uniform disclosure form (available at http://dx.doi. org/10.21037/apm-20-1093). The authors have no conflicts of interest to declare.

Ethical Statement: The authors are accountable for all aspects of the work in ensuring that questions related to the accuracy or integrity of any part of the work are appropriately investigated and resolved. All patients signed informed content. The study was conducted in accordance with the Declaration of Helsinki (as revised in 2013) and was approved by the ethics committee of our hospital (Lot. 20140704).

Open Access Statement: This is an Open Access article distributed in accordance with the Creative Commons Attribution-NonCommercial-NoDerivs 4.0 International License (CC BY-NC-ND 4.0), which permits the noncommercial replication and distribution of the article with the strict proviso that no changes or edits are made and the original work is properly cited (including links to both the formal publication through the relevant DOI and the license). See: https://creativecommons.org/licenses/by-nc-nd/4.0/.

\section{References}

1. Singer M, Deutschman CS, Seymour CW, et al. The third internationalconsensus definitions for sepsis and septic shock (sepsis-3). JAMA 2016;315:801-10.

2. Walley KR. Sepsis-induced myocardial dysfunction. Curr Opin Crit Care 2018;24:292-9.

3. Kakihana Y, Ito T, Nakahara M, et al. Sepsis-induced myocardial dysfunction: pathophysiology and management. J Intensive Care 2016;4:22.

4. Brodska H, Valenta J, Pelinkova K, et al. Diagnostic and prognostic value of presepsin vs. established biomarkers in critically ill patients with sepsis or systemic inflammatory response syndrome. Clin Chem Lab Med 2018;56:658-68.

5. Ackland GL, Prowle JR. Presepsin: solving a soluble (CD14) problem in sepsis? Intensive Care Med 2015;41:351-3.

6. Ehrman RR, Sullivan AN, Favot MJ, et al. Pathophysiology, echocardiographic evaluation, biomarker findings, and prognostic implications of septic cardiomyopathy: a review of the literature. Crit Care 2018;22:112.

7. Havaldar AA. Evaluation of sepsis induced cardiac dysfunction as a predictor of mortality. Cardiovasc Ultrasound 2018;16:31.

8. McDonough KH,Virag JI. Sepsis-induced myocardial dysfunction and myocardial protection from ischemia/ reperfusion injury. Front Biosci 2006;11:23-32.

9. Mehta S, Gill SE. Improving clinical outcomes in sepsis and multiple organ dysfunction through precision medicine. J Thorac Dis 2019;11:21-8.

10. Fattahi F, Ward PA. Complement and sepsis-induced heart dysfunction. Mol Immunol 2017;84:57-64.

11. Tomita K, Takashina M, Mizuno N, et al. Cardiacfibroblasts: contributory role in septic cardiac 
dysfunction. J Surg Res 2015;193:874-87.

12. Sato R, Nasu M. A review of sepsis-induced cardiomyopathy. J Intensive Care 2015;3:48.

13. Joseph LC, Kokkinaki D, Valenti MC, et al. Inhibition of NADPH oxidase 2 (NOX2) prevents sepsis-induced cardiomyopathy by improving calcium handling and mitochondrial function. JCI Insight 2017;2:e94248.

14. Yang F, Zhao LN, Sun Y, et al. Levosimendan as a new force in the treatment of sepsis-induced cardiomyopathy: mechanism and clinical application. J Int Med Res 2019;47:1817-28.

15. Zangrillo A, Putzu A, Monaco F, et al. Levosimendan reduces mortality in patients with severe sepsis and septic shock: A meta-analysis of randomized trials. J Crit Care 2015;30:908-13.

16. Gordon AC, Perkins GD, Singer M, et al. Levosimendan for the Prevention of Acute Organ Dysfunction in Sepsis. N Engl J Med 2016;375:1638-48.

17. Antcliffe DB, Santhakumaran S, Orme RML, et al. Levosimendan in septic shock in patients with biochemical evidence of cardiac dysfunction: a subgroup analysis of the LeoPARDS randomised trial. Intensive Care Med 2019;45:1392-400.

Cite this article as: Liu J, Dai M, Yang H, Song L, Chen K, Wang Y, Hua M. Serum level of soluble CD14 subtype predicts long-term prognosis in sepsis patients with cardiac dysfunction. Ann Palliat Med 2020;9(4):2054-2061. doi: 10.21037/apm-20-1093
18. Jeong HS, Lee TH, Bang CH, et al. Risk factors and outcomes of sepsis-induced myocardial dysfunction and stress-induced cardiomyopathy in sepsis or septic shock: A comparative retrospective study. Medicine (Baltimore) 2018;97:e0263.

19. Shahreyar M, Fahhoum R, Akinseye O, et al. Severe sepsis and cardiac arrhythmias. Ann Transl Med 2018;6:6.

20. Shozushima T, Takahashi G, Matsumoto N, et al. Usefulness of presepsin (sCD14-ST) measurements as a marker for the diagnosis and severity of sepsis that satisfied diagnostic criteriaof systemic inflammatory response syndrome. J Infect Chemother 2011;17:764-9.

21. Zhang X, Liu D, Liu YN, et al. The accuracy of presepsin (sCD14-ST) for the diagnosis of sepsis inadults: a metaanalysis. Crit Care 2015;19:323.

22. Yang HS, Hur M, Yi A, et al. Prognostic value of presepsin in adult patients with sepsis: Systematic review and metaanalysis. PLoS One 2018;13:e0191486.

23. Matera G, Quirino A, Peronace C, et al. Soluble CD14 Subtype-A New Biomarker in Predicting the Outcome of Critically Ill Septic Patients. Am J Med Sci 2017;353:543-51. 